\title{
Evolution of Secondary Electrons Emission During EUV Exposure in Photoresists
}

\author{
Roberto Fallica ${ }^{1, *}$, Stefano Nannarone ${ }^{2}$, Nicola Mahne $^{2}$, Andrea Marco Malvezzi ${ }^{2}$, \\ Andrea Berti ${ }^{2}$, Danilo De Simone ${ }^{1}$ \\ ${ }^{1}$ IMEC, Leuven, Belgium \\ ${ }^{2}$ CNR-IOM Trieste, Italy \\ *roberto.fallica@imec.be
}

\begin{abstract}
Low energy electronic processes are key to chemical reactions during exposure of photoresists in extreme ultraviolet lithography. To understand and optimize the functionality of photoresists, it is of paramount important to assess the magnitude of the electron distribution inside the material. While photoemission spectroscopy is being widely used, vacuum barrier crossing and nonideal photoemissivity alter the measured spectra in a way that prevents meaningful assessment of very low energy electrons. In this work we propose a model to account for the physics of photoemission effects and to reliably estimate the distribution from solid state matter. The model also provides a quantitative value for the occupation function and density of state. We tested the model on the photoemission spectra acquired on a prototype EUV photoresist and report here the results.

Keywords: EUV lithography, Electronic processes, Secondary electrons, Photoemission, Photoresist, Photoelectron spectroscopy
\end{abstract}

\section{Introduction}

Recent experimental evidence indicates that scattering processes involving electrons of very low kinetic energy $<<10 \mathrm{eV}$ are the main driver for chemical reactions in organic molecules [1]. Specific examples including electron scattering studies on isolated molecules in gas phase [2,3] and their role in desorption mechanisms [4] indicate how electronic processes triggered by low energy $(\sim$ $\mathrm{eV})$ electrons are at the base of most lithographically relevant phenomena. For electron attachment and dissociation where a molecule is taken in the scattering process into an unstable excited state with modified electronic and structural configuration resulting in fragmentation and desorption.

Low-energy electron-induced reactions have substantial technological importance in Extreme Ultra-Violet (EUV) optical lithography which uses light beams with photons of energy well above the ionization threshold and smaller wavelengths that allow a jump in the high-resolution patterning of thin organic polymer-based photoresist films. Contrary to previous optical lithography technologies such as deep ultraviolet (DUV), in EUV the amount of incident photons is about one order of magnitude lower than it was when UV or longer wavelength photons were used. As a result, the exposure mechanism that leads to the lithographic process is ascribed to the electron cascade generated by the primary photoelectron filling up the formerly empty electronic states in absence of illumination more than the photoelectron creation itself.

Photoresists (PR) for EUV lithography consist of, as in previous technology, an organic polymer backbone plus small percentages of photoacid generator (PAG) and acid quencher to catalyze or slow down the deprotection reaction, respectively. These compounds are therefore known as chemically amplified resists (CAR) since their invention [5]. Due to the nature of EUV light and electronic structure of photoresist material, photoabsorption mostly involves electrons energetically located in the valence band occupying molecular orbitals involving atomic $\mathrm{O}, \mathrm{C}$ and $\mathrm{H}$ states whose excitation triggers the production of primary, highenergy electrons [6].

$\begin{array}{llr}\text { Received } & \text { April } & 8,2021 \\ \text { Accepted } & \text { April } & 22,2021\end{array}$


In case of PR made up of atomic species with electron binding energies lower than photon energies photo-electrons due to core level excitation and related Auger electrons have to be added to this scenario, a case which is not at issue here.

Owing to the electron mean free path of photoelectrons, most of the kinetic energy and momentum of primaries is transferred to PR through multiple inelastic electron-electron scattering events in sub-picosecond timescale, which generate a substantially larger amount of electrons bond to occupy low energy electronic states [8]. All this experimental evidence leads to the conclusion that low energy electrons are the main responsible in starting chemical reactions in the photoresist at variance with the early expectations. For lithography engineers, the amount and distribution of secondary electrons in photoresists during exposure to EUV light is therefore key to assess the efficiency of the litho process.

Though it is not yet entirely quantitatively assessed the way these electronic processes translate into lithographic performance (conventionally described in terms of figures of merit such as sensitivity, roughness, resolution [9], and defectivity). Present contribution is devoted to the presentation of an experimental method and the model it stems from aims at establishing a protocol to assess the total amount of low energy electron production and their distribution among electronic states inside the PR during light exposure. Experimentally, the PR is exposed to the working photon energy of $\hbar \omega=92 \mathrm{eV}$ and the distribution in kinetic energy of emitted electrons per incident photon $N_{\text {ext }}\left(E_{k}\right)$ is measured in the region spanning from the electron emission onset of secondary electrons up to a few tens of eV. The $N_{\text {ext }}\left(E_{k}\right)$ distribution is modeled in terms of PR parameters and the figure of merit of the rate of production of low energy electrons expressed by an out-ofequilibrium occupation function $f^{*}(E)$, formerly empty in absence of illumination.

Furthermore, it is worth to stress that the distribution in kinetic energy and momentum of the emitted electrons is deformed in energy, $E$, and momentum caused by the PR-vacuum barrier height $\chi$, resulting in a net energy change, and reduction of the normal component of momentum and consequent electron diffraction. Because of the barrier cutoff, the low energy end of electron states with energies lower than $\chi$ is experimentally inaccessible, a limitation which is overcome performing a suitable extrapolation of the occupation function obtained for the electron energy above the barrier range. We believe that this procedure provides a reliable estimation of the energy distribution of electrons inside the photoresist when the material is under illumination in the light patterning phase of the photolithographic process. The underlying assumptions are discussed in light of the known literature of photoemission from organic molecules in the solid-state layers and some examples are presented.

\section{Experimental}

Low energy electron emission yield studies of EUV photoresists were carried out by electron yield spectroscopy at the CNR-IOM synchrotron beamline BEAR [10] (Elettra, Trieste-Italy) using a photon beam of EUV light $(\lambda=13.5 \mathrm{~nm}$ wavelength, $\hbar \omega=91.9 \mathrm{eV})$ of $\approx 200 \mu \mathrm{m}^{2}$ cross section area whose intensity was calibrated by an absolute photodiode AXUV-100. In this apparatus, samples were loaded through an interlock onto a motorized stage located in a vacuum chamber which had been evacuated at a base pressure in the $10^{-10} \mathrm{mbar}$ range for the entire duration of the experiment. The distribution $N_{\text {ext }}\left(E_{k}\right)$ in kinetic energy of electrons emitted from the sample surface was measured using an hemispherical electron analyzer with resolution $\Delta E$ $=0.1 \mathrm{eV}$ positioned in normal emission with the light impinging at $45^{\circ}$ in $s$-polarization incidence During the measurement, the samples were also biased to a voltage of $-30 \mathrm{~V}$ (kinetic electrons energy where obtained rescaling by $30 \mathrm{eV}$ ) to get an almost parallel electron beam in the region of fly in vacuum and then to maximize the electron trajectories collected by the electron analyzer. Kinetic energy distribution of emitted electrons was then acquired in the very low energy range starting from the secondary emission onset and covering a kinetic energy range $\sim 15 \mathrm{eV}$.

Photoresist samples consisted of a EUVpatternable blend of polymer (82.7 wt.\%) and photoacid generator (PAG) (17.3 wt.\%). The polymer was poly(hydroxystyrene) (PHS) modified with 50\% t-Butyloxycarbonyl (tBOC) protecting group, $\mathrm{C}_{16} \mathrm{O}_{3} \mathrm{H}_{18}$. The $\mathrm{PAG}$ was a triphenylsulfonium salt, $\mathrm{C}_{23} \mathrm{O}_{3} \mathrm{H}_{17} \mathrm{~S}_{2} \mathrm{~F}_{9}$. Molecular structures are shown in Figure 1. 

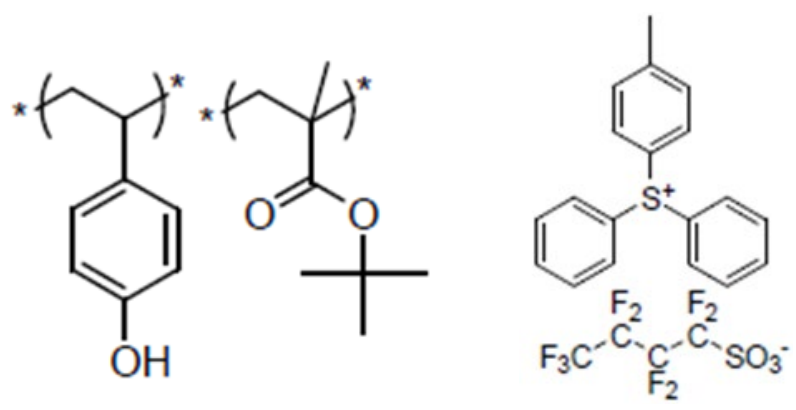

Fig. 1. Structure of the photoresist investigated, consisting of a poly(hydroxystyrene) backbone with tButyloxycarbonyl protecting groups (left) and a triphenylsulfonium salt as photoacid generator (right).

The material was spin coated on blank silicon wafers of 4" diameter, then baked at $100{ }^{\circ} \mathrm{C}$ for 60 $\mathrm{s}$ on a hot plate to remove the remaining solvent. By tuning the spin coating speed, uniform film of $35 \pm$ $1 \mathrm{~nm}$ thickness was obtained by ellipsometry. The silicon substrate was then cleaved into $2 \times 2 \mathrm{~cm}^{2}$ dices and kept in low vacuum until their loading in the vacuum chamber.

The measurement procedure consists of a first measurement of sample in pristine condition ('unexposed'). Count rate was obtained sitting with the light spot at a fixed surface point with an acquisition time of $1 \mathrm{~s}$ which resulted in $\sim 2 \mathrm{~s}$ of illumination per kinetic energy point corresponding to a dose $\sim 1 \mathrm{~mJ} / \mathrm{cm}^{2}$ per kinetic energy point resulting in a total dose $\sim 10 \mathrm{~mJ} / \mathrm{cm}^{2}$ for 10 points.

Best effort was taken to minimize the sample exposure which, according to the photon flux and duration of the acquisition, is estimated to be $\approx 0.7$ $\mathrm{mJ} / \mathrm{cm}^{2}$ (much lower than the dose to clear and thus negligible from the point of view of the chemical changes induced). Afterwards, the sample was also measured after having been exposed to increasing amount of EUV light and comparable to the typical dose-to-size for this class of photoresists.

\section{Analytical model of outside versus inside electron energy distribution}

An essential account of the model is given here, referring the reader to a future publication for details. First, the model accounts for the crossing of the vacuum barrier which makes the electron lose part of their energy, $E$, and momentum component along the normal to the surface, $z$, with subsequent refraction. The zero of energy distribution inside the material is taken as the bottom of the conduction band or LUMO (Lowest Unoccupied Molecular Orbital,) according to the terminology of solids or molecular aggregates (similarly, valence band top is reported as HOMO - Highest Occupied Molecular Orbital). The vacuum level is taken as zero of kinetic energy of electrons emitted in vacuo according to the relation:

$$
E=E_{K}+\chi
$$

The material is assumed to be in stationary conditions at an out of equilibrium temperature $T^{*}$. The occupation of states by charge carriers is described by an occupation function $f^{*}\left(T^{*}\right)$.

The physical parameters in the model are the position of the quasi-Fermi level inside the gap, $E_{F}$, the electronic affinity, $\chi$, and the temperature $T^{*}$ of the electron gas in the conduction band which is as first trial entry assumed as a free electron gas with a density of states $g(E) \propto \sqrt{ } E$, as supported by previous theoretical result [11].

The $N_{\text {ext }}\left(E_{k}\right)$ distribution of emitted electrons results modeled through an integral on the $z$ coordinate normal to the surface on the portion of light excited and out of equilibrium portion of material of a combination of functions describing the light attenuation, electron transport to the surface and barrier crossing at its turn integrated on the polar angle between electron momentum and

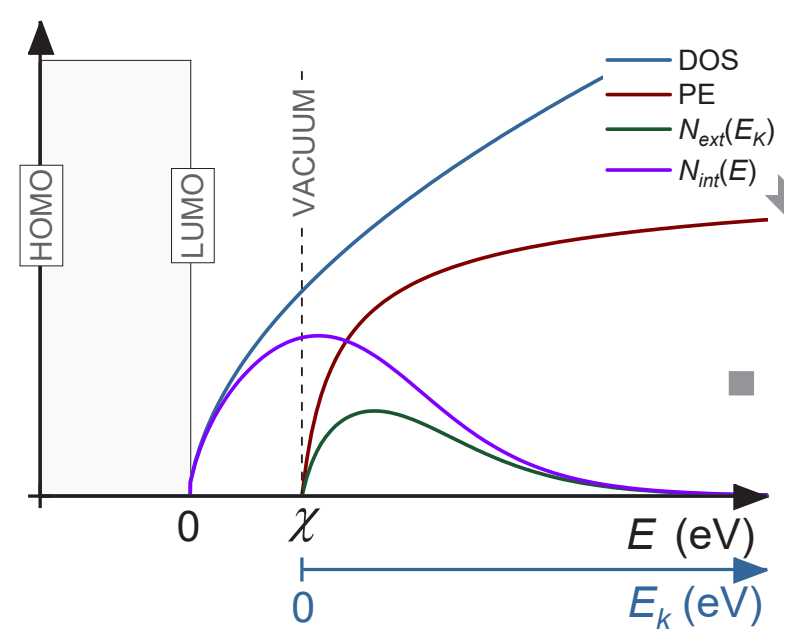

Fig. 2. Plot of the functions involved in the analytical model for photoemission. HOMO: highest occupied molecular orbital and top of the valence band. LUMO: lowest unoccupied molecular orbital and bottom of the conduction band. HOMO and LUMO are separated by a bandgap. Vacuum level is aligned to the electronic affinity $\chi$ (i.e., the barrier for photoemission). $E$ is the energy of carriers inside the material. $E_{k}$ is the kinetic energy of photoemitted photoelectrons. The electronic distribution inside the material, $N_{i n t}(E)$ is determined by density of states (DOS) and occupation function $\left(f^{*}(E)\right.$, not shown). The distribution of photoemitted electrons is $N_{\text {ext }}\left(E_{k}\right)$ and it is a function of internal distribution in the photoresist $N_{\text {int }}(E)$ and of the photoemission model PE. 
surface normal with the rate of emission at a given kinetic energy band weighted by the occupation function $f^{*}$. The functions involved in the model are depicted Fig. 2. It should be noted that the model does not require the modeling of the dynamics of the cascade of secondary electrons being just devoted to inferring from the experimental secondary emission at what extent the low electron energy levels in the material become occupied through the electron cascade mechanism.

\section{Results}

The experimental distribution $N_{\text {ext }}\left(E_{k}\right)$ of photoelectrons emitted from a polymer + PAG photoresist film as a function of the kinetic energy $E_{K}$ is shown in Fig. 3a. The spectrum presents the typical overall shape of secondary electron emission with a slowly decaying tail for $E_{K}>4 \mathrm{eV}$ and a peak approaching the kinetic energy zero. The electron affinity in model calculation is assumed to be $\chi=2$ $\mathrm{eV}$ and as a result the LUMO and band gap are highlighted at $E_{K} \leq-\chi$.

As entry value for the quasi-Fermi level, the work using a linear assumption behavior of material work function using the value $E_{F}=-2.5 \mathrm{eV}$ with respect to the LUMO level. Using a minimization routine to fit to the experimental $N_{\text {ext }}\left(E_{k}\right)$, the temperature $T^{*}$ of the electron gas was found to be $T^{*}=15073 \mathrm{~K}$. However, we could not find a satisfactory fit in the very low kinetic energy portion of the spectrum as evidenced by comparing the fit function dashed line in Figure 3a with experiment. This disagreement can be ascribed to a deviation of the density of states from that of the free electron gas because of the presence of localized molecular orbital(s) close to the vacuum level. The large electron mean free path of low $E$ electrons can also be responsible of overweighting the contribution of emission from low energy states or even from substrate. This effect, ignored in the model, might imply an electron yield higher that PR.

Under the hypothesis of localized molecular orbital(s), an additional term $\delta(E)$ to the density of states $g(E)$ can be introduced to take this extra emission into account. The $f^{*}(E)$ function presented here is obtained with this correction to the free electron gas density of states. This calls for specific theoretical calculations of density of states of empty orbitals in this energy range to validate or reject this kind of hypothesis.

In summary, the fitting function in the thermal approximation seems to be able to provide insight into the occupation of states in the conduction band close to energy bottom in the region from LUMO up to a few eV above vacuum level. Provided the $g(E)$ from theory or experiment, the amount of the electronic states of the photoresist that are occupied during the stationary exposure to the EUV beam can be estimated, as shown in Figure $3 b$.

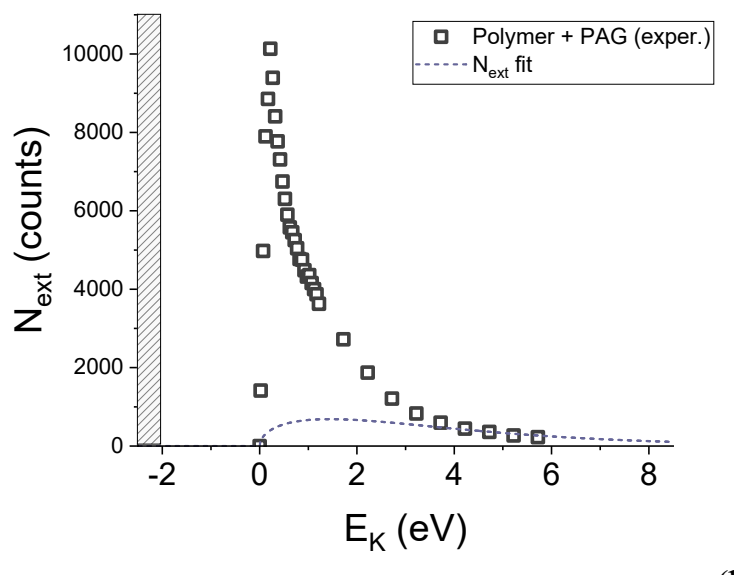

(b)

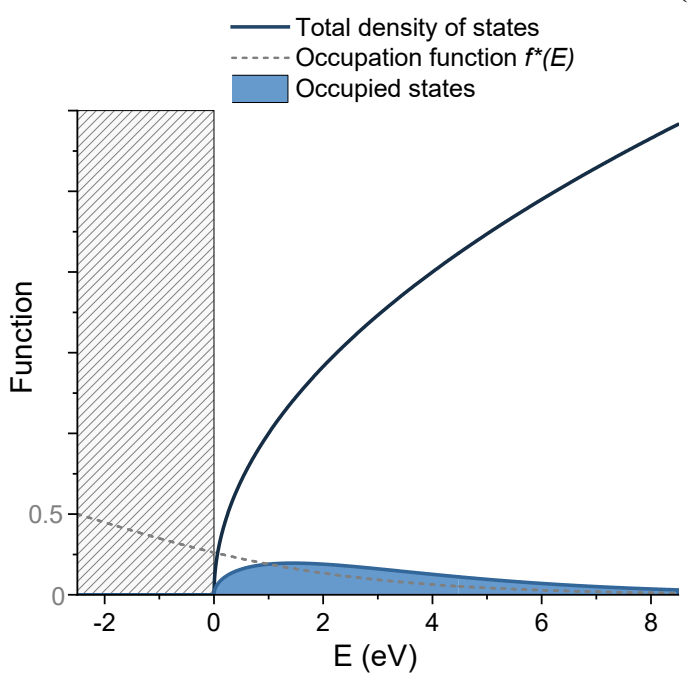

Fig. 3. (a) Experimental photoemission data (black squares) and model fitting (dashed blue line). (b) Total density of states (dark blue line), occupation function $f^{*}(E)$ (dashed yellow line) and resulting occupied states in the conduction band of the photoresist (shaded light blue area). The quasi-Fermi level is located at $E_{F}=-2.5$ $\mathrm{eV}$, and the vacuum level is located at $E=\chi=2 \mathrm{eV}$. The highlighted region below $E=0 \mathrm{eV}$ (LUMO) is the bandgap.

\section{Conclusions}

In this work we present an analytical model to determine the inner distribution of electrons in a material during exposure to EUV light from the experimental secondary electron distribution in kinetic energy close to the emission onset. Our 
approach gives insight into the occupation of low energy electronic states in the region spanning from the LUMO and extrapolated data and up to states in the vacuum level region.

Although there are aspects to understand and refine, we believe that this method shows promising validity and represents an example of how one can experimentally determine and monitor the distribution of very low energy electrons generated in a photoresist under stationary EUV radiation exposure conditions in the photoresist which have energies below the vacuum threshold, i.e., that cannot be measured experimentally. This model provides for the first time a complete picture of very low energy electrons, which are relevant for lithographic purposes.

\section{Acknowledgements}

Photoresist manufacturers are kindly acknowledged for the providing test materials. The authors also wish to thank Vasiliki Kosma and Ivan Pollentier (imec) for fruitful discussions. Stimulating discussion with J. Rezvani are acknowledged, A. Giglia and K. Koshmak are thanked for their support in data acquisition.

\section{References}

1. "Low-Energy Electrons: Fundamentals and Applications" edited by Oddur Ingólfsson, Jenny Stanford Publishing; 1st edition (2019). ISBN13: 978-9814800006.

2. R. M. Thorman, R. K. T. P., D. H. Fairbrother, and O. Ingólfsson, Beilstein J. Nanotechnol., 6 (2015) 1904.
3. I. I. Fabrikant, S. Eden, N. J. Mason, and J. Fedor, Advances In Atomic, Molecular, and Optical Physics, 66 (2017) 545.

4. M. Bertin, I. Martin, F. Duvernay, P. Theule, J. B. Bossa, F. Borget, E. Illenberger, A. Lafosse, T. Chiavassa, and R. Azria, Phys. Chem. Chem. Phys., 11 (2009) 1838.

5. H. Ito, C. G. Willson, and J. M. J. Frechet, Digest of Technical Papers of 1982 Symposium on VLSI Technology (1982) 86.

6. A. Narasimhan et al., J. Micro/Nanolith. MEMS MOEMS 14(4) (2015) 043502.

7. R. Fallica, S. J. Rezvani, S. Nannarone, S. Borisov, D. De Simone, S. Babin, G. Lorusso, and G. Vandenberghe, Proc. SPIE, 10960 (2019) 1096009.

8. R. E. Chase, W. L. Gordon, and R.W. Hoffman, Applications of Surface Science, 4 (1980) 271.

9. G. M. Gallatin, P. Naulleau, D. Niakoula, R. Brainard, E. Hassanein, R. Matyi, et al., Proc SPIE, 6921 (2008) 69211E.

10.S. Nannarone, F. Borgatti, A. DeLuisa, B. P. Doyle, G. C. Gazzadi, A. Giglia, P. Finetti, N. Mahne, L. Pasquali, M. Pedio, G. Selvaggi, G. Naletto, M. G. Pelizzo, and G. Tondello, AIP Conference Proceedings 705(1) (2004) 450. http://www.elettra.trieste.it/elettrabeamlines/bear.html.

11.S. Serra, E. Tosatti, S. Iarlori, S. Scandolo, and G. Santoro, Phys. Rev. B, 62, (2000) 7. 